\title{
La désindustrialisation : quelles réalités dans le cas français?
}

Deindustrialization: what are the realities concerning the French case?

Deindustrialisierung: Welche Realitäten im französischen Fall ?

\section{François Bost et Dalila Messaoudi}

\section{(2)enEdition}

Journals

Édition électronique

URL : http://journals.openedition.org/rge/6333

ISSN : 2108-6478

Éditeur

Association des géographes de l'Est

Référence électronique

François Bost et Dalila Messaoudi, «La désindustrialisation : quelles réalités dans le cas français ? », Revue Géographique de l'Est [En ligne], vol.57 / 1-2 | 2017, mis en ligne le 15 novembre 2017, consulté le 08 septembre 2020. URL : http://journals.openedition.org/rge/6333

Ce document a été généré automatiquement le 8 septembre 2020

Tous droits réservés 


\title{
La désindustrialisation : quelles réalités dans le cas français?
}

\author{
Deindustrialization: what are the realities concerning the French case? \\ Deindustrialisierung: Welche Realitäten im französischen Fall?
}

François Bost et Dalila Messaoudi

\section{Introduction}

1 L'industrie française employait 3,1 millions de personnes en 2015, soit environ $12 \%$ de l'emploi total, auxquels s'ajoutaient 4,5 millions d'emplois indirects. Ses 260000 entreprises (dont $90 \%$ de PME et de TPE) représentaient à cette date une valeur ajoutée de 274 milliards d'euros (dont 219 milliards pour le seul secteur manufacturier), soit $12,4 \%$ de la VA nationale (10 \% pour le secteur manufacturier). Principale contributrice des exportations de la France ( $74 \%$ du total, soit 445 milliards d'euros), elle est aussi le secteur d'activité qui investit le plus dans la recherche \& développement nationale, soit 25 milliards d'euros.

2 En dépit de ces chiffres, qui témoignent de son indéniable rôle moteur dans l'économie française, l'industrie est depuis plusieurs années confrontée à un mouvement de désindustrialisation rapide et important, dont la prise de conscience est récente. Souvent présentée comme un phénomène inéluctable, cette dynamique commence à inquiéter l'opinion et les décideurs en raison des menaces de "perte de substance économique » (Fontagné et Lorenzi, 2005), voire de "déclin » qu'elle fait peser sur l'ensemble de l'économie nationale.

3 L'intérêt que suscite désormais la désindustrialisation l'a élevée au rang de véritable objet d'étude, notamment chez les géographes, dont certains ont réorienté en partie leurs travaux pour réinvestir les questions industrielles nationales - qui ne suscitaient alors plus guère d'intérêt - sous des angles d'approches largement renouvelées.

4 Si la définition de la désindustrialisation ne pose aucune difficulté de compréhension (soit la « réduction du nombre d'emplois dans le secteur industriel d'un pays, de même que celle 
du secteur de l'industrie par rapport aux autres secteurs d'activité " selon le dictionnaire Larousse), en revanche sa mesure statistique pose certains problèmes importants à connaître pour en nuancer l'analyse. Cet article entend donc s'interroger sur la perception, la mesure statistique, de même que la signification réelle des chiffres censés rendre compte de la désindustrialisation à l'échelle nationale. Après avoir analysé la lente prise de conscience de ce phénomène et de ses menaces économiques et sociales (I), l'étude passera au crible ses principaux indicateurs et leur portée exacte (II). Enfin, elle dressera un tableau de la désindustrialisation à l'échelle des 304 zones d'emplois (III).

\section{Entre sous-estimation et déni de la réalité}

\section{A. Un phénomène longtemps sous-estimé...}

5 Omniprésent depuis plusieurs années dans les médias français, le terme de «désindustrialisation» est paradoxalement absent des dictionnaires de géographie francophones, de même que des quelques manuels et lexiques de géographie économique, tous déjà anciens il est vrai. Au mieux celui-ci était-il employé incidemment dans les publications, mais sans pour autant avoir été élevé au rang d'objet géographique.

6 Ce contraste saisissant ne traduit en rien le désintérêt des géographes français pour ce sujet désormais important et anxiogène, surtout si l'on en juge par les travaux très récents qui ont depuis largement abordé ce phénomène, notamment à la faveur de la mise au programme entre 2014 et 2016 des concours du CAPES et de l'agrégation de la question des mutations des systèmes productifs en France (Bost, 2015, Carroué, 2014 ; Dugot et Thuillier 2014 ; Fache et Baudelle, 2015 etc.). En fait, jusqu'à la fin des années 2000, les travaux des géographes (au même titre d'ailleurs que ceux des économistes, des institutions comme la DATAR, ou encore les rapports parlementaires) préféraient lui substituer des termes renvoyant à des dynamiques de changement profond (donc plus optimistes), à l'instar de ceux de « reconversion » ou de « mutations » industrielles (Nesta, 2010) en vogue depuis les années 1970. La « désindustrialisation » était d'ailleurs encore communément qualifiée de «mythe » ou de «fantasme » dans certains rapports ministériels ou parlementaires (Roustan, 2004), mais aussi dans la presse économique durant la décennie 2000... Pour autant, les observateurs les plus clairvoyants n'en faisaient pas moins état d'une certaine inquiétude pour l'avenir. En 2005, par exemple, les économistes L. Fontagné et J.-H. Lorenzi n'hésitaient pas à parler de « risque de perte de substance économique » à propos de la désindustrialisation.

7 Il faut donc que la situation se soit singulièrement dégradée ces toutes dernières années dans l'industrie française pour que le seuil de perception d'une réalité que l'on ne voulait pas voir soit enfin franchi, au point que certains spécialistes plus clairvoyants aient pu commencer à parler d'« emballement", de "choc " (Lorenzi, 2014) ou de « décrochage» (Gallois, 2012; Giraud et Weil, 2013; Cohen et Buigues, 2013).

8 A cet égard, les sérieuses difficultés rencontrées depuis plus d'une dizaine d'années par le secteur automobile français ont joué un rôle de révélateur décisif dans cette prise de conscience salutaire (Messaoudi, 2015). Entre 2004 et 2013, la production de véhicules particuliers fabriquée sur le sol français a ainsi chuté de près de 50 \% passant de 3,2 
millions à 1,7 million de véhicules. En 2016, la France n'était plus que le onzième producteur mondial et le troisième producteur européen ${ }^{1}$ (après l'Allemagne et l'Espagne), alors qu'elle se plaçait au quatrième rang mondial et au second rang européen en 2000. Les baisses des productions hexagonales ont eu comme conséquence directe un effondrement des effectifs industriels. Entre 2000 et 2014, selon les données du Comité des Constructeurs Français d'Automobiles (CCFA), la production réalisée dans l'hexagone par les deux grands constructeurs français, Renault et PSA, a fortement régressé (- $40 \%)$ et près de 100000 emplois ont été détruits dans ce secteur (Bost 2014). En 2016, le secteur comptait dans son ensemble près de 220000 salariés (dont $9 \%$ dans l'assemblage de véhicules et $36 \%$ dans la fabrication d'équipements automobiles, le reste étant réparti dans les activités de fabrication de carrosseries et de remorques, etc.), contre 321000 salariés en 2000 (INSEE). Les inquiétudes pèsent désormais sur la pérennité de plusieurs sites de production français. L'ouverture et la mise en concurrence des territoires ont donc bouleversé le paysage automobile français. Or, le devenir de cette industrie est un enjeu majeur pour les territoires à toutes les échelles.

\section{B. ...et plus ancien qu'on le croit}

9 A rebours des idées reçues, la désindustrialisation n'est pas une nouveauté en France puisque ce phénomène était déjà l'œuvre dès la seconde moitié des années 1970, sans pour autant avoir donné lieu à un discours spécifique et alarmant (Bost, 2012). Il était alors d'usage de n'y voir qu'une conséquence naturelle de la productivité du travail. Identifié par Jean Fourastié dès 1949, le glissement de l'emploi industriel vers le secteur des services faisait alors écho à celui déjà observé de l'emploi agricole vers l'industrie durant la Révolution industrielle (Verlet, 1997 ; Feiertag, 2011). C'est cette spectaculaire tertiarisation de l'emploi dans les pays développés qui est d'ailleurs à l'origine du succès de l'expression de "société post-industrielle " créée par les sociologues Alain Touraine en France dès 1969 et Daniel Bell aux Etats-Unis en 1973. Les deux fondateurs du courant sociologique dit "post-industrialiste " entendaient dénommer ainsi l'ère nouvelle dans laquelle entraient tous les pays développés dans les années 1970. Cette expression exerça une influence considérable sur l'opinion, les hommes politiques et les décideurs, au point de laisser penser - à tort a posteriori - que l'industrie n'était plus aussi essentielle pour la puissance et le rayonnement d'un Etat.

Cette mutation profonde du système productif fut accompagnée par la puissance publique, principalement par la requalification des salariés concernés et le soutien (notamment via la DATAR) des vieilles régions industrialisées frappées de plein fouet par la crise des années 1970 (Nord-Pas-de-Calais, nord de la Lorraine, Moselle, vallées vosgiennes, Ardenne, Alsace, bassin stéphanois, région lyonnaise, bassin du Creusot, Alpes du nord, Basse Loire, vallée de la Seine, etc.), en encourageant avec des succès très inégaux leurs efforts de mutation par l'implantation d'activités à plus forte valeur ajoutée et à plus fort contenu de $\mathrm{R} \& \mathrm{D}$.

11 Les premiers effets de la désindustrialisation ont commencé à être plus clairement perceptibles à partir du milieu des années 1980, à la faveur de la concurrence exercée par les pays à bas salaires (on ne parlait pas encore de pays " émergents »). L'abandon des activités à fort coefficient de main-d'œuvre et à faible contenu technologique (textile-habillement et chaussure dans le bas de gamme par exemple) est alors apparu 
comme inéluctable, à moins de les délocaliser vers l'Europe du sud, l'Afrique du nord ou certains pays asiatiques et l'opinion s'est faite plus fataliste à leur égard (Messaoudi, 2012).

12 La spectaculaire montée en puissance des pays émergents d'Asie orientale, d'Amérique latine ou d'Europe orientale au cours des années 1990-2000 a encore aggravé la situation, car elle a poussé les entreprises industrielles françaises, au même titre que celles des autres pays industrialisés, à réorganiser leur production sur une base mondiale (sinon à l'échelle des zones d'intégration régionale comme l'Union européenne, l'ALENA, le MERCOSUR, etc.) pour faire face à cette concurrence imprévue par son ampleur et sa rapidité, mais aussi pour profiter de nouvelles opportunités de croissance (Bost, 2012 et 2014). L'accélération du processus de désindustrialisation est ainsi apparue comme l'une des conséquences de l'ouverture tous azimuts des pays industrialisés au Monde, dont le corollaire fut la mise en concurrence globale et inédite des salariés les uns avec les autres. Le nombre des salariés est en effet passé de 1,5 milliard dans le monde au début des années 1980 à plus de 3 milliards en 2010.

Mais jusqu'au milieu des années 2000, les conclusions des grandes études commanditées par l'Etat français sur la question de la désindustrialisation demeuraient cependant encore nuancées et prudentes. La responsabilité des pays émergents dans la désindustrialisation était encore fortement minorée. Le ministère de l'Industrie estimait par exemple sa part dans les pertes d'emplois industriels à seulement $13 \%$ en 2004.

\section{La désindustrialisation et ses différents indicateurs : une réalité désormais tangible}

\section{Le « décrochage » au prisme de l'effondrement de l'emploi industriel et de la contribution de l'industrie au PIB}

La publication en 2012 du rapport intitulé «Pacte pour la compétitivité de l'industrie française », remis au Premier ministre par le comité d'experts dirigé par Louis Gallois, a fait office d'électrochoc national, puisque pour la première fois le diagnostic faisait explicitement le constat du "décrochage" de l'industrie française. Les auteurs n'hésitaient plus à tirer la sonnette d'alarme : "l'industrie française atteint aujourd'hui un seuil critique, au-delà duquel elle est menacée de déstructuration » (Gallois, 2012: 9). Le lien entre la désindustrialisation et le manque de compétitivité de l'économie y était également démontré. Surtout, à l'instar de plusieurs travaux qui l'ont suivi (Giraud et Weil 2013 notamment), le rapport Gallois officialisait que les pays émergents (la Chine en particulier) et à plus bas salaires d'une manière générale jouaient désormais un rôle plus important que par le passé dans la destruction des emplois industriels en France.

Le graphique 1, élaboré à partir des séries longues de l'INSEE, illustre en valeur absolue l'effondrement continu des effectifs de l'industrie française (salariés et non-salariés, à temps plein ou pas) entre 1970 (5,6 millions) et 2014 (3,3 millions), soit une baisse de 2,3 millions (la baisse annuelle moyenne s'élevant à environ 52200 personnes), sachant que l'emploi industriel a connu historiquement son plus haut niveau en France en 1973, avec 5959000 personnes (ce sommet a été atteint au milieu des années 1960 au Royaume-Uni et au milieu des années 1950 aux Etats-Unis). 
En valeur relative, les effectifs dans l'industrie sont ainsi passés de $23,7 \%$ à $12 \%$ de la population active à peine entre 1970 et 2014. Mais le graphique témoigne cependant du caractère non linéaire de cet effondrement, deux paliers pouvant être identifiés au tournant des années 1990 et 2000. La crise de 2007-2008 en a encore aggravé le mouvement cependant.

Graphique 1 : Evolution des effectifs de l'industrie française entre 1970 et 2014 (en milliers)

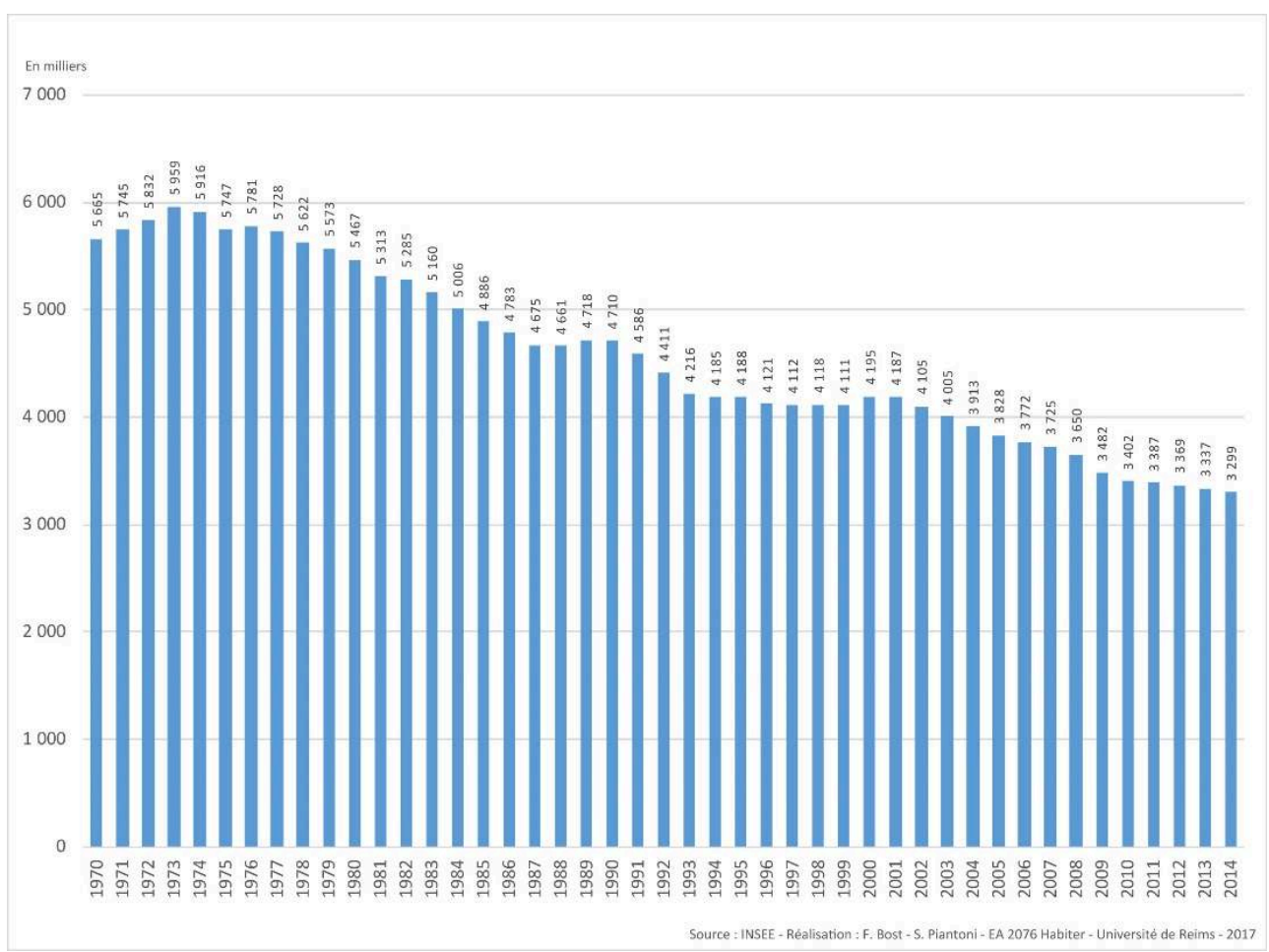

En comparaison avec d'autres pays développés, le cas de la France est loin d'être isolé (cf. tableau 1). Ainsi, sur la période 1991-2010, la France, l'Allemagne et le Japon ont affiché une baisse de l'emploi manufacturier importante et assez proche en valeur relative (respectivement $-30,7 \%,-28,7 \%$ et $-32,3 \%$ ). L'effondrement est encore plus spectaculaire au Royaume-Uni (- $41 \%)$. La baisse est en revanche plus contenue dans le cas des Etats-Unis (- 28,7 \%, à égalité avec l'Allemagne) et surtout de l'Italie (-16\%) et de l'Espagne (- 9,1\%).

Tableau 1 : Evolution de l'emploi dans l'industrie manufacturière dans quelques grands pays développés sur la période 1991-2010 (en \%)

\begin{tabular}{|l|l|}
\hline Pays & Baisse de l'emploi en \% (par ordre décroissant) \\
\hline Royaume-Uni & $-41,1 \%$ \\
\hline Japon & $-32,3 \%$ \\
\hline Allemagne & $-30,7 \%$ \\
\hline Etats-Unis & $-28,7 \%$ \\
\hline
\end{tabular}




\begin{tabular}{|l|l|}
\hline France & $\mathbf{- 2 8 , 6} \%$ \\
\hline Suède & $-24,2 \%$ \\
\hline Italie & $-16,7 \%$ \\
\hline Espagne & $-9,1 \%$ \\
\hline
\end{tabular}

source : base de données AMECO, Commission européenne

Quant à la contribution de l'industrie spécifiquement manufacturière au PIB marchand national (cf. graphique 2), elle n'était plus que de $10 \%$ en 2016 en France (26,3 \% en $1978 ; 14,1 \%$ en 2000), contre $20,3 \%$ en Allemagne, ce qui témoigne de son décrochage impressionnant avec le voisin d'outre-Rhin - dont elle a été longtemps le challenger -, où ce secteur reste une valeur sûre. Plus inquiétant, la France se situe désormais aussi en retrait par rapport à des pays comme l'Italie (14,2 \%) et l'Espagne $(12,1 \%)$ qui étaient il y a peu encore derrière elle. Maigre consolation, elle ne devance plus que celle du Royaume-Uni (8,7\%) parmi les grands pays de la zone.

En revanche, la contribution de l'industrie manufacturière de la France au PIB de l'Union européenne (10,8\% en 2016) la place sensiblement au même niveau que celles de l'Italie (11,4 \%) et du Royaume-Uni (11 \%), l'Espagne se situant encore loin derrière $(6,4 \%)$ selon les chiffres de l'agence EUROSTAT. En dehors de l'Allemagne, qui fait toujours figure de locomotive ( $30,2 \%$, soit trois fois plus que la France), tous ces pays se situent bien loin de l'objectif de $20 \%$ du PIB qui avait été fixé pour cette activité par le Comité des Régions de l'UE au début des années 2000.

Graphique 2 : Place de l'industrie manufacturière dans quelques pays représentatifs de l'UE en 2016

\section{Un phénomène dont il convient cependant de relativiser la portée}

Les deux indicateurs les plus couramment utilisés pour parler de la désindustrialisation (l'évolution de l'emploi industriel et de la contribution de l'industrie au PIB) sont-ils pour autant les plus pertinents pour mesurer ce phénomène ? En effet, depuis sa montée en épingle dans les médias et les discours politiques, un certain nombre d'observateurs ont fait valoir que, contrairement aux apparences, et sans pour autant le remettre fondamentalement en cause, la désindustrialisation n'était pas aussi forte qu'on pourrait le croire, notamment en raison de certains artefacts statistiques (Bost, 2014).

21 L'effondrement de l'emploi industriel en France peut ainsi être réexaminé à l'aune de ses trois principaux déterminants, tels qu'ils ont été identifiés dans l'étude de référence de Lilas Demmou (Direction générale du Trésor), intitulée La désindustrialisation en France (Demmou, 2010) :

- le premier de ces déterminants est celui de la concurrence par les coûts en provenance des pays en développement, mais aussi, fait plus nouveau, des pays développés eux-mêmes, notamment membres de l'Union européenne (cette origine souligne l'aggravation du différentiel de compétitivité avec les pays voisins, tout particulièrement l'Allemagne, l'Espagne, l'Italie ou encore le Royaume-Uni), qui représente à elle seule environ $30 \% \mathrm{du}$ 
total des pertes d'emplois industriels en France depuis 2000. Le manque structurel de compétitivité de l'industrie française revêt différents aspects bien connus que l'on citera simplement pour mémoire, en particulier :

- les coûts salariaux élevés de la main-d'œuvre : le coût horaire moyen de la France se situe en 2017 au quatrième rang européen, derrière ceux de la Belgique, de la Suède et du Danemark. Pourtant l'industrie ces deux derniers pays ne semble pas en pâtir particulièrement et cette question n'y fait pas débat comme en France, sans doute en raison de leur positionnement sectoriel plus orienté vers le haut de gamme. Il apparaît cependant inenvisageable en France de baisser le niveau des salaires moyens pour y suppléer, ce qui invite plus que jamais au renforcement de la productivité du travail et à l'accélération de la robotisation des tâches les plus répétitives et les moins qualifiées ;

- la fiscalité appliquée aux entreprises françaises, qui est la seconde la plus élevée d'Europe (avec un taux moyen de 33,33 \% en 2017), après celle de la Belgique (33,99\%), alors que l'impôt sur les sociétés s'élève à 12,5\% en Irlande, $20 \%$ au Royaume-Uni (mais $17 \%$ annoncé d'ici à 2020), $22 \%$ au Danemark, $25 \%$ en Espagne et $30 \%$ en Allemagne, la moyenne de l'Union européenne se situant autour de $25 \%$. Conscient de ce handicap pour l'attractivité de la France (tant auprès des investisseurs étrangers que des investisseurs nationaux potentiels), le Président Macron a annoncé en mai 2017 qu'il ferait de ce point l'une des priorités de son quinquennat (avec un abaissement séquentiel, annoncé en août 2017, qui devrait porter l'impôt sur les sociétés à $25 \%$ en 2022, soit le niveau moyen actuel de l'Union européenne);

- ou encore les lourdeurs évidentes de l'actuel Code du travail qui ne facilitent pas la fluidité du marché de l'emploi à la différence d'autres pays, notamment en Europe du nord (flexisécurité par exemple au Danemark), où les entreprises craignent beaucoup moins d'embaucher. La réforme en profondeur du Code du travail français a d'ailleurs été érigée en priorité des mesures du quinquennat de Président Macron en 2017, au point d'être imposée par ordonnances afin d'en accélérer la mise en œuvre.

Les deux autres déterminants de l'effondrement de l'emploi industriel en France selon L. Demmou se répartissaient à peu près à égalité quant aux pertes d'emplois entre :

- la hausse constante de la productivité du travail : l'observatoire français des conjonctures économiques (OFCE) l'a évalué à 3,6 \% en rythme annuel pour le secteur manufacturier durant la période 1996-2006, tandis que celle-ci s'établissait à 1,2 \% pour l'ensemble de l'économie,

- enfin, l'externalisation d'une multitude d'activités transférées par les entreprises industrielles à des sociétés de services industriels et d'intérim : entretien des équipements, gardiennage, ménage, sécurité, restauration collective, intendance informatique, bureaux d'études, conseil juridique, publicité, marketing, comptabilité, paie, services après-vente, logistique, etc. Ces activités de services aux entreprises industrielles étaient en effet jusquelà comptabilisées en emplois industriels du simple fait que la maison mère relevait du secteur secondaire. Signe de ce glissement massif, leurs effectifs ont été multipliés par quatre en quarante ans. Avec un chiffre évalué à 4 millions d'emplois, ils dépassent désormais en valeur absolue ceux du secteur industriel. Le recours croissant des entreprises industrielles au travail intérimaire (enregistré par définition dans les «services aux entreprises", même si leurs missions relèvent de l'industrie) contribue aussi fortement à cette baisse statistique de l'emploi industriel.

La ventilation statistique des principales causes de la désindustrialisation permet au final de les classer en deux grandes familles. La première renvoie aux causes logiques et 
mécaniques : glissement statistique de nombreux emplois depuis le secteur secondaire vers le secteur tertiaire (externalisation de certaines activités), mais sans destruction nette d'emplois, puisque ceux-ci sont transférés et même démultipliés dans le secteur des services, qui est leur place naturelle; hausse de la productivité du travail dans l'industrie (non remplacement des emplois après départ à la retraite; transfert des salariés vers d'autres tâches ; licenciements dans certains cas). De ce fait, celles-ci n'ont rien d'anormal.

La seconde famille correspond en revanche à la facette la plus médiatique, la plus spectaculaire et inquiétante qui soit : celle des destructions nettes d'emplois, suite à la faillite de nombreuses entreprises, à la fermeture d'usines ou à la cessation de lignes de production. A cet égard, il est important de rappeler que ces emplois détruits sous les effets de la " concurrence par les coûts » (soit environ un tiers des emplois industriels disparus chaque année selon l'étude de L. Demmou), c'est-à-dire par manque de compétitivité et délocalisations diverses, sont ceux qui relèvent explicitement de la désindustrialisation.

Aussi est-il possible d'estimer, à la suite de l'économiste Philippe Askenazy, que le terme de désindustrialisation est sans doute «un peu fort, de même que l'attention médiatique qui lui est accordée » (Askenazy, 2012 : 38).

Au final, ce rapide décryptage semble indiquer que l'évolution de l'emploi industriel sur le temps long n'est sans doute pas le meilleur indicateur pour apprécier à sa juste valeur le phénomène contemporain de désindustrialisation. Il est en effet dans la logique des choses que les progrès technologiques continuent à détruire encore des milliers emplois par les progrès de la productivité, notamment les moins qualifiés, qui sont les plus faciles à automatiser (mécanisation, robotisation). Il paraît vain de vouloir inverser radicalement cette tendance. D'autre part, l'organisation de la production va poursuivre sa profonde transformation amorcée dans les années 1980 et ce, au détriment de l'emploi industriel. D'une structure verticale fondée sur l'intégration en interne de toutes les activités (depuis la conception jusqu'aux services après-vente), les entreprises industrielles se sont en effet reconfigurées de manière horizontale suite à l'externalisation de leurs activités non stratégiques. Cette évolution est plus que jamais perçue comme la condition sine qua non de l'accroissement de leur compétitivité (voire de leur survie !) et l'on voit mal comment revenir en arrière.

Aussi la désindustrialisation contemporaine doit-elle être passée au crible d'autres indicateurs, qui permettent d'identifier par ailleurs les causes profondes de ce phénomène: évolution des marchés à l'export et de la balance des paiements, positionnements sectoriels, $\mathrm{R} \& \mathrm{D}$, taille des entreprises, etc.

\section{D'autres indicateurs pertinents à mobiliser pour appréhender l'ampleur et la gravité de la désindustrialisation}

Largement excédentaire jusqu'en 1976 pour les biens de consommation courante, la balance commerciale de la France est devenue déficitaire à partir de 2000, tout particulièrement pour les biens intermédiaires (automobile, électronique et informatique, matériels de transport, etc.) qui tiraient jusque-là les exportations. C'est à partir de 2003 que la balance commerciale est devenue structurellement négative, suite aux importations de plus en plus massives de biens intermédiaires et de consommation en provenance de l'étranger (notamment des pays à bas coûts, mais pas 
seulement), mais aussi de produits énergétiques dont les prix se sont littéralement envolés de 2002 à 2007. Le déficit commercial français s'est ainsi établi à - 48,1 milliards d'euros en 2016. Celui-ci est cependant en diminution régulière par rapport aux années précédentes (- 67,2 milliards d'euros en 2012; et surtout - 74,2 milliards d'euros en 2011, record historique) qui ont été catastrophiques. Mais cette amélioration récente des termes de l'échange est surtout liée aux effets conjoints de la baisse du coût des hydrocarbures sur le marché mondial ainsi qu'à la dépréciation du cours de l'euro plus qu'à une évolution structurelle de l'industrie française à l'export.

Si cette évolution de la balance commerciale montre que la France consomme plus qu'elle ne produit, celle-ci est aussi une conséquence directe de la désindustrialisation (Giraud et Weil, 2013), suite à la disparition d'entreprises françaises ou de certaines de leurs activités de production, dont les biens vendus en France ou exportés jusque-là ont pu être remplacés par des importations depuis l'étranger (Bost, 2014). Elle témoigne surtout pour les entreprises restantes de leur perte structurelle de compétitivité, sachant que la balance commerciale se compose à l'export et en valeur pour les troisquarts de biens industriels, le solde se composant de services (finance, tourisme, etc.). Cette situation, qui révèle la faiblesse de l'industrie française et son affaiblissement graduel, n'est pas récente, mais elle s'est aggravée depuis une quinzaine d'années, au point de s'affirmer comme une évidence.

$\mathrm{Au}$ total, si la perte de compétitivité de l'industrie française est bien l'une des principales causes de la désindustrialisation (notamment face à la concurrence étrangère qui a fait perdre de nombreux marchés domestiques), elle explique aussi très largement sa rétrogradation à l'export, dans un contexte inédit où le nombre de pays exportateurs concurrents n'a jamais été aussi important, surtout parmi les pays émergents. Ainsi la France ne représentait plus que 3,1\% des exportations mondiales en 2014 selon le Rapport de l'OMC (ce qui la classait au $8^{\mathrm{e}}$ rang mondial à cette date), contre 4,7 \% en 2000 et encore plus de $6 \%$ dans les années 1970 et 1980. De surcroît, la part de marché de la France à l'exportation vers l'Union européenne, son premier débouché (avec 59,8\% en 2016, dont 46,4 \% rien que pour la zone euro, loin devant l' Asie,12,7 \%, les Amériques, 10,9\%, et 7,2 \% pour l'Europe hors UE) a aussi baissé significativement, passant de $12,7 \%$ à 10,5\% entre 2000 et 2014 .

31 La baisse progressive des parts de marché des entreprises françaises à l'exportation constitue donc un facteur inquiétant pour leur avenir sur le sol national. Nombre d'observateurs insistent en effet sur le rôle essentiel des exportations (notamment celles en direction des pays développés et émergents) en matière de création d'emplois dans les pays industrialisés. Or les exportations industrielles françaises sont trop peu diversifiées pour atteindre pleinement cet objectif, si bien qu'elles dépendent dangereusement de quelques secteurs clés comme l'aéronautique et l'agroalimentaire (respectivement premier et second postes excédentaires), qui occupent l'un et l'autre le second rang mondial dans leur domaine; mais aussi, selon les années, le matériel militaire (avec les succès retentissants et récents des avions rafale du groupe Dassault, ou encore les sous-marins de la DCNS), de transport, les produits pharmaceutiques, les parfums et cosmétiques, etc.

32 De surcroît, et contrairement à certaines idées reçues qui survalorisent le secteur des hautes technologies dans les représentations de la puissance, le positionnement sectoriel de l'industrie française demeure encore trop marqué par le bas et le moyen de gamme, qui peine à exporter, où les marges bénéficiaires sont faibles et continuent 
même à se réduire pour faire face à la concurrence. De plus en plus intenable, cette situation limite, ou ne permet plus, le réinvestissement dans la $\mathrm{R} \& \mathrm{D}$ et la modernisation de l'appareil productif, conditions élémentaires pour monter en gamme. La plupart des produits français entrent alors frontalement en concurrence avec ceux fabriqués sur ces mêmes segments par les pays émergents qui se sont lancés avec succès dans ces activités, mais de manière plus compétitive (Bost, 2012 et 2014). A l'inverse, la balance commerciale de l'Allemagne (+ 297 milliards de dollars en 2016, soit l'excédent des comptes courants le plus élevé au monde, devant celui de la Chine, avec + 245 milliards de dollars) tire profit de manière exceptionnelle de la grande diversification de ses productions et surtout de son positionnement dans les secteurs du moyen et du haut de gamme (sans parler de l'incomparable réputation de qualité de ses produits!). C'est ce choix stratégique qui lui permet de pratiquer des marges bénéficiaires élevées et une politique de réinvestissement systématique dans la $R$ \& $D$ et la modernisation de son outil de production pour conserver une avance qui lui vaut de figurer au second rang mondial des puissances exportatrices derrière la Chine, mais devant les Etats-Unis et le Japon.

Face à un solde commercial structurellement déficitaire, qui pousse à s'endetter davantage chaque année pour maintenir un niveau de consommation équivalent, la France dispose de peu d'alternatives pour inverser la tendance et revenir ne serait-ce qu'à une situation d'équilibre :

-réduire les importations (les biens industriels représentant $83 \%$ de leur valeur en 2015) : cette hypothèse apparaît peu envisageable à court et moyen terme, eu égard au fait que nombre de biens industriels et technologiques ne sont guère substituables par des produits nationaux qui ne peuvent plus être produits en France pour des raisons de coûts des facteurs, mais aussi parce que les importations d'hydrocarbures sont appelées à grever encore le déficit,

--substituer des services aux exportations de biens industriels défaillantes: vraie dans l'absolu, en vertu de la théorie des avantages comparatifs et du discours sur la "société post-industrielle » qui annonce le passage d'une économie industrielle à une économie de services, cette option se révèle a posteriori bien décevante car elle ne peut compenser le déficit industriel et énergétique. En effet, la plus grande partie de la valeur ajoutée créé par les services se réalise par le biais des filiales des firmes françaises implantées directement à l'étranger (réseaux des banques françaises à l'étranger, enseignes de la grande distribution, etc.), donc localement, et non par l'exportation de ces mêmes services (Giraud et Weil, 2013). Les répercussions de ces activités sur la balance des paiements se révèlent alors faibles. Très concurrencés aussi à l'export par des pays plus compétitifs, les services n'ont réalisé en 2016 qu'un excédent des plus modestes (0,4 milliard d'euros) dans la balance commerciale française. Enfin la contribution des services plafonnent aujourd'hui autour de $20 \%$ du total des exportations mondiales, ce qui témoigne du rôle toujours irremplaçable de l'industrie dans la dynamique des exportations,

- -finalement, n'en déplaise aux tenants de la « société post-industrielle », l'industrie demeure et demeurera pour longtemps encore le moteur du commerce entre les pays. Aussi est-ce dans un tandem industrie / services orienté dans la production de biens exportables (ou «nomades », pour reprendre l'expression proposée par P.-N. Giraud et T. Weil) et engagé résolument dans une démarche de montée en gamme que la France peut espérer résorber son déficit commercial. 

consenti en $\mathrm{R} \& \mathrm{D}$, donc à l'innovation et ce depuis de nombreuses années. La France est en effet encore loin du seuil annuel des $3 \%$ de PIB dans la $R \& D$ ainsi qu'y invitait dès 2000 l'agenda de Lisbonne dans le cadre européen (injonction renouvelée en 2010 par l'adoption de la Stratégie Europe 2020) : 2,21 \% du PIB en 2016 (valeur qui a très peu évolué depuis 1996), contre $2,87 \%$ en Allemagne, où ces dépenses ont par contre grimpé de $75 \%$ entre 2005 et 2016 . Ce handicap majeur empêche fondamentalement l'immense majorité des entreprises industrielles de monter en gamme et donc de proposer des produits nouveaux, à fortes marges, susceptibles d'être réinvesties dans la R \& D. Cette situation est encore aggravée par un «effet taille »: la France manque en effet d'entreprises moyennes et de taille intermédiaire, à la différence de l'Allemagne. Or ce sont d'abord celles-ci qui tirent l'emploi et les exportations, et au final investissent le plus dans la R \& D (hors grands groupes). Aussi lorsqu'elles investissent, l'immense majorité des entreprises françaises se concentrent en priorité sur le simple renouvellement de leurs équipements, au détriment des investissements dits de rupture technologique.

insuffisance de la R \& D et de l'innovation est étroitement corrélée au médiocre positionnement sectoriel des entreprises industrielles françaises, qui est favorable à la désindustrialisation. Ainsi, la surreprésentation de l'emploi industriel dans les secteurs de basse et de moyenne-basse technologies (66\%) selon la nomenclature d'activités françaises (NAF rév. 1) est porteuse de beaucoup de destructions d'emplois, car c'est bien dans ces domaines que l'on trouve en premier lieu les fonctions les plus menacées par l'accroissement de la productivité, de même que les faillites potentielles d'entreprises. La part de l'emploi industriel dans les secteurs des moyennes-hautes technologies $(24 \%)$ et surtout des hautes technologies $(10 \%)$ est donc encore trop faible en France. C'est pourtant dans ces domaines que les pouvoirs publics voient le salut de l'industrie française. C'est d'ailleurs ce constat qui a motivé la mise en œuvre des pôles de compétitivité en 2005 (au nombre de 71 en 2017).

Très liée à la question de l'innovation, celle de la modernisation des équipements constitue un autre indicateur très utile à mobiliser pour appréhender l'exposition des entreprises à la désindustrialisation.

Celle-ci peut se mesurer tout d'abord par le suivi de l'âge moyen du parc des machines qui ne cesse d'augmenter, ce qui témoigne d'un renouvellement très insuffisant. En 2013, date de la dernière étude disponible (SYMOP-GIMELEC-DGE), ce parc avait en moyenne 19 ans d'âge, contre 17,5 ans en 1998. C'est l'une des raisons qui explique notamment pourquoi le taux d'utilisation des machines était en moyenne de $81 \%$ en France (machines en panne ou trop vétustes pour être pleinement utilisées, réduction des volumes produits ne justifiant pas de nouveaux investissements), contre $95 \%$ en Allemagne. En 2014, 34 \% des industriels français estimaient que leur outil de production était peu compétitif selon une étude du cabinet Roland Berger.

Mais c'est le degré de robotisation des usines qui focalise le plus l'attention ces dernières années, à la fois comme mesure de l'un des talons d'Achille de l'industrie française (par son insuffisance, reflet du sous-investissement chronique), mais aussi comme solution prometteuse pour sauver l'industrie. Menée elle aussi par le cabinet Roland Berger, la dernière étude disponible sur le sujet insiste sur le différentiel abyssal exprimé en valeur absolue entre la France (34 000 robots à peine) et l'Allemagne (162 000) en 2014, sans qu'il soit actuellement possible d'expliquer clairement cette situation 
(l'hypothèse actuellement avancée étant que le patronat français serait, de par sa sociologie, plus rétif au progrès). Néanmoins, rapportée au nombre d'actifs dans l'industrie, la situation apparaît moins catastrophique, puisqu'il s'élevait à 84 robots pour 10000 employés en France, contre 125 outre-Rhin. Mais plus que sur leur nombre total, c'est surtout sur leur très inégale répartition intersectorielle qu'il convient d'insister. C'est de très loin l'industrie automobile qui a le plus fortement recours à la robotisation des tâches en France, puisque l'on y compte 695 robots industriels pour 10 000 employés, contre 755 en Allemagne, soit un écart de $9 \%$, ce qui est moins élevé que ce que l'on pouvait penser a priori. Cependant, en valeur absolue, la différence reste absolument spectaculaire entre les deux pays, puisque ces parcs de robots s'élevaient respectivement dans ce secteur à 18000 contre 86000 unités... Rappelons que le nombre d'usines dans le secteur automobile allemand est sans commune mesure avec celui de la France. Plus inquiétante en revanche est la situation observée dans les autres secteurs industriels, principalement dans l'agroalimentaire et l'industrie des métaux, où le taux de robotisation français présente des écarts notoires avec l'Allemagne (respectivement $55 \%$ et $85 \%$ ). L'écart est encore plus grand dans le secteur de la chimie-plasturgie, où il s'établissait à $129 \%$. La dynamique de réindustrialisation des pays développés, mais aussi la baisse des coûts unitaires de ces machines devrait cependant voir exploser leurs usages, selon une étude du Boston Consulting Group, qui estime à $10 \%$ sa progression annuelle mondiale pour les dix prochaines années.

A côté des indicateurs classiques investigués pour mieux cerner la désindustrialisation figurent d'autres indicateurs possibles, le plus intéressant étant certainement celui proposé depuis 2009 par l'institut de veille économique Trendéo à partir d'informations collectées par différents canaux. Désormais souvent cité et relayé par la presse économique, cet indicateur révèle que jusqu'en 2015 le nombre de fermetures a excédé largement celui des ouvertures : 1775 fermetures contre 1178 créations, soit un solde de 597 usines. En 2016, pour la première fois depuis la création de cet indicateur, fermetures et ouvertures sont à égalité (136 chacune), ce qui a fait dire un peu vite et malencontreusement aux médias que l'industrie française avait enfin cessé son hémorragie. Il n'empêche, cet indicateur est à suivre. Selon Trendéo, les fermetures sont surtout le fait de petits établissements (71 employés en moyenne). Fait symptomatique, les nouvelles usines sont minuscules en termes d'emplois créés (une vingtaine de salariés seulement en moyenne lors de leur ouverture en 2016 et ce chiffre n'a cessé de baisser depuis 2009), ce qui bouleverse les représentations en matière d'«usine type ». Il faut y voir un effet direct de leur robotisation et de leurs investissements dans les technologies, impératifs seuls à même d'assurer leur pérennité à moyen et long terme. Evidemment, ces établissements peuvent être amenés à embaucher davantage par la suite, au gré de leur développement.

Tableau 2 : Evolution des fermetures et des ouvertures d'usines en France (2009-2016)

\begin{tabular}{|l|l|l|l|l|l|l|l|l|}
\hline & $\mathbf{2 0 0 9}$ & $\mathbf{2 0 1 0}$ & $\mathbf{2 0 1 1}$ & $\mathbf{2 0 1 2}$ & $\mathbf{2 0 1 3}$ & $\mathbf{2 0 1 4}$ & $\mathbf{2 0 1 5}$ & $\mathbf{2 0 1 6}$ \\
\hline Fermetures & 380 & 261 & 190 & 266 & 266 & 220 & 192 & 136 \\
\hline Ouvertures & 156 & 226 & 156 & 177 & 127 & 177 & 159 & 136 \\
\hline
\end{tabular}




\begin{tabular}{|l|l|l|l|l|l|l|l|l|}
\hline Solde & -224 & -261 & -34 & -89 & -140 & -43 & -33 & 0 \\
\hline
\end{tabular}

source : Trendéo

\section{Des zones d'emplois diversement concernées}

41 La mesure de la désindustrialisation au niveau national ne nous dit rien sur la réalité de ce phénomène aux échelles fines des territoires, qui sont généralement peu abordées par les économistes. Celle des «zones d'emplois", telles que définies par l'INSEE (" Espaces à l'intérieur desquels la plupart des actifs résident et travaillent, et dans lesquels les établissements peuvent trouver l'essentiel de la main-d'cuvre nécessaire pour occuper les emplois offerts »), est sans nul doute la plus pertinente d'un point de vue géographique pour répondre à cette préoccupation, puisque leur vocation est précisément de pouvoir donner une vue d'ensemble des statistiques de l'emploi et du chômage sur un territoire donné. De surcroît, leur découpage présente le grand intérêt de transcender les unités administratives (départements et régions), qui se révèlent souvent mal adaptées pour apprécier la réalité.

Les zones d'emplois ont été identifiées en 1983-1984 à partir de l'étude des données de déplacements domicile-travail collectées lors des recensements de la population. Leurs périmètres ont été révisés en 2010 afin de tenir compte des changements récents affectant l'espace français : évolution du rayonnement des villes les polarisant; baisse de l'activité dans les zones d'emplois; allongement des distances domicile / travail ; etc.

43 Au final, les 304 zones d'emplois de la France métropolitaine présentent de vigoureux contrastes en termes de taille, de population et d'emplois, qui reflètent la diversité de leur poids économique, leur attractivité très différente, leurs spécificités de localisation dans l'espace national, l'influence ou non d'une métropole dans leur aire ou à proximité, etc.

44 Les données de l'INSEE disponibles couvrent en détail la période 1998-2014, soit 16 années, ce qui est tout de même suffisant pour apprécier la diffusion de la désindustrialisation à l'échelle du pays et poser la question de son aggravation à la faveur de la crise financière et économique apparue en 2007-2008.

\section{Rares sont les bassins non concernés par la désindustrialisation}

La carte 1, intitulée "Gain et pertes d'emplois industriels par zones d'emplois (1998-2014) », donne une image de la vigueur de la désindustrialisation entre ces deux dates. Sur les 304 zones d'emplois métropolitaines, 252 enregistrent des pertes nettes (soit 83 \%) durant la période, celles-ci allant de - 110445 à Paris (la zone d'emplois de Paris compte 121 communes, dont les 20 arrondissements de Paris ${ }^{2}$; elle a chuté de 326 362 en 2014 à 215917 emplois en 1998), à - 4 emplois à Loudéac. Compte tenu de leur spécialisation ancienne, c'est dans les vieilles régions industrialisées, c'est-à-dire celles qui ont connu la Révolution industrielle, que s'enregistrent les plus fortes baisses: région parisienne et Picardie (- 23419 emplois notamment dans la zone d'emplois de Roissy-Sud Picardie), Nord-Pas-de-Calais (dont - 17648 et - 17530 dans les zones d'emplois de Roubaix-Tourcoing et de Lille), Alsace (- 15477 et - 13183 dans les zones 
d'emplois de Mulhouse et de Strasbourg), porte de Bourgogne (- 18143 dans la zone d'emplois de Belfort-Montbéliard-Héricourt), Champagne-Ardenne (dont - 12998 pour la seule zone d'emplois de Troyes ; - 6511 dans celle de Reims), Lorraine (- 7442 et - 7 369 dans les zones d'emplois de Nancy et de Metz), régions lyonnaise (- 27 977) et stéphanoise (- 17444 ), vallée de la Seine jusqu'à son aval (dont - 14652 et - 7418 dans les zones d'emplois de Rouen et du Havre). La coalescence des cercles suggère à cet égard, que ce sont bien des régions entières qui se sont désindustrialisées et pas seulement des villes. La carte 2, intitulée « Evolution de l'emploi industriel par zones d'emplois (1998-2014) » montre de surcroît que la vitesse de ce phénomène est rapide, les pertes atteignant même en moyenne entre $-2 \%$ et $-3,5 \%$ / an dans plusieurs zones d'emplois hautement représentatives: Belfort-Montbéliard-Héricourt, Roissy-sud de Picardie, Lens, Hénin, Roubaix-Tourcoing, Flandre-Lys, Saint-Quentin, Longwy, Thionville, Troyes, Vitry-le François / Saint-Dizier, Châlons-en-Champagne, Evreux, etc. Plus généralement, les pertes s'échelonnent ailleurs entre $-1,9 \%$ et $-0,5 \% /$ an durant la période 1998-2014.

Le mouvement de désindustrialisation a commencé à l'occasion de la crise économique mondiale des années 1970 et de ses effets dans les bassins industriels construits sur l'exploitation et la transformation des matières premières (charbon, fer, etc.), mais aussi sur l'industrie manufacturière (textile, construction mécanique, etc.). En raison de leurs coûts d'exploitation élevés et de la faiblesse de leur rentabilité, les établissements ont été fermés les uns après les autres dans les années 1980, ou alors maintenus artificiellement à l'aide de subventions publiques. Le caractère monoindustriel - voire mono-établissement - de certaines villes explique que des régions entières ont été frappées avec une très grande violence par une crise à la fois économique, sociale (chômage chronique) et urbaine (dégradation du bâti, fermeture de nombreux commerces, etc.) qui a fait durablement de leurs bassins d'emplois des zones d'évitement vis-à-vis des activités nouvelles et porteuses de développement. Leurs handicaps sont alors devenus comme autant de défis complexes à relever pour leur transformation : vétusté et inadaptation des infrastructures ; formation insuffisante de la main-d'œuvre (notamment dans les technologies nouvelles) ; paysages très dégradés (friches industrielles, corons désaffectés, pollution des sols et des eaux courantes, etc.) ; aménités jugées peu attractives ; etc.

L'entrée en crise simultanée de ces zones d'emplois explique le changement d'approche de la politique de l'Etat qui, d'un rôle d'aménageur, est passé à celui de "pompier » cherchant à éteindre tous les incendies. D'abord en favorisant l'arrivée de nouvelles activités par le biais d'incitations fiscales et de primes à l'embauche (Bost, 2014); puis en investissant dans l'amélioration des infrastructures de transport et la formation professionnelle (enseignement technologique notamment), ou encore en aidant à la réhabilitation de nombreuses friches industrielles. Dans un second temps (années 1990-2000), car plus lentes à démarrer, les initiatives locales ont conjugué aussi leurs efforts. Mais la reprise de la désindustrialisation, notamment à la faveur de la crise de 2007-2008 et de ses suites, a freiné bien des efforts de mutation dans ces régions fragiles et convalescentes, si bien que les poches de pauvreté et de sous-emploi restent nombreuses aujourd'hui dans toutes ces régions. Leur situation apparaît d'autant plus délicate que ces régions ont moins bénéficié que les autres des relais de croissance générés ailleurs en France par le secteur public et les activités dites résidentielles (Davezies, 2012). 

désindustrialisation est en effet un phénomène qui atteste de l'obsolescence de la classique diagonale reliant Le Havre à Marseille et séparant la France en deux moitiés (la France des régions industrielles au nord-est; la France des villes industrielles au sud-ouest), qui continue de marquer les représentations mentales concernant l'industrie. A l'exception du quart sud de la France et partiellement de l'ouest, où l'industrie paraît résister et ne pas trop perdre d'effectifs, presque toutes les régions sont en fait concernées à des degrés divers par la désindustrialisation, mais à des rythmes de progression variables (cf. carte 2). Ce constat témoigne de la diffusion de l'industrie dans un nombre plus important de régions françaises, et ce depuis le milieu $\mathrm{du} \mathrm{XX}^{\circ}$ siècle.

nt donc de réserver une attention particulière à ces régions que l'on pourrait qualifier de périphériques par rapport aux bastions traditionnels de l'industrie en France. La petitesse des cercles sur la carte 1 s'y explique surtout par la plus faible présence d'emplois industriels dans ces territoires (il y a donc moins d'emplois à détruire), et non par une plus faible désindustrialisation (cette dernière peut y être $a$ priori importante en proportion dans certaines zones d'emplois). Une étude plus poussée sur ce sujet devra s'attacher à faire le lien entre la désindustrialisation et les secteurs d'activité touchés par ce phénomène, dans la mesure où ces régions présentent d'autres spécificités par rapport aux bastions hérités de la Révolution industrielle. De surcroît, nombre de ces zones d'emplois paraissent éloignées des métropoles et de leurs effets d'entraînement sur le marché du travail. Elles pâtissent enfin de la surreprésentation des petites et moyennes villes (notamment en milieu rural), moins attractives par rapport aux nouveaux facteurs de localisation des activités industrielles. Cependant, la principale différence entre ces régions périphériques moins industrialisées et le cœur industriel de la France au nord et à l'est est que l'impact de la désindustrialisation y est néanmoins amorti par la dynamique beaucoup plus forte des activités administratives (les emplois publics ont notamment continués à y croître, ce qui ne se vérifie moins dans les régions anciennement industrialisées), mais aussi résidentielles et touristiques (Le Blanc, 2011 et 2012 ; Davezies, 2012).

\section{Les gains d'emplois industriels restent l'exception}

La zone d'emplois de Toulouse en Occitanie (carte 1), par ailleurs la plus vaste zone d'emplois de la métropole avec 717 communes, est la seule à se singulariser en France par sa progression très vigoureuse en valeur absolue entre les deux dates de référence (+ 12575 emplois), grâce en particulier au dynamisme du secteur aéronautique et spatial, dont dépendent aussi de nombreux centres de R \& D (leurs effectifs sont comptabilisés au titre de l'industrie lorsqu'ils relèvent de groupes industriels), ainsi qu'un très important réseau d'entreprises sous-traitantes. Au palmarès, Toulouse est suivie par la zone d'emplois de La Roche-sur-Yon (100 communes en Vendée), qui enregistre un gain de +4206 emplois. Plus loin encore, figurent 5 zones d'emplois (par ordre décroissant: Figeac, Saint-Nazaire, Sablé-sur-Sarthe, Toulon et Vitré), qui enregistrent des progressions allant de + 1207 emplois industriels (Figeac, en Occitanie), à + 1027 (Vitré, en Bretagne). Trois d'entre elles (hors Saint-Nazaire et Toulon) relèvent de petites villes et de territoires plutôt ruraux, bien éloignés des métropoles, mais où le secteur manufacturier est ancien, bien ancré localement et appuyé sur une solide tradition ouvrière et sociale, caractéristiques partagées d'ailleurs 
par La Roche-sur-Yon. La zone d'emplois de Figeac (76 communes dans le département du Lot) totalise notamment un emploi sur 5 dans l'industrie (21\%), soit deux fois plus que la moyenne nationale, alors qu'elle rencontre des problèmes d'accessibilité. Les secteurs industriels de ces différentes zones d'emplois sont assez diversifiés, mais ils relèvent le plus souvent (hors Toulouse) de secteurs traditionnels (mécanique, construction navale, agro-alimentaire, meuble, etc.) ayant avantageusement tirés profit d'effets de spécialisation (systèmes productifs locaux), mais aussi de mutations technologiques et organisationnelles réussies. Pour autant, les hautes technologies n'y sont pas absentes, comme à Figeac, où la société Figeac Aero (implantée depuis 1989; 1600 emplois en 2016) est devenue un des sous-traitants leaders en France dans la mécanique de précision pour le secteur de l'aviation (pièces aéronautiques en alliages légers et métaux durs). Sa nouvelle usine en cours de construction à Figeac devrait d'ailleurs être l'une des plus modernes d'Europe dans son domaine.

Dans les 42 autres zones d'emplois en progression (ouest et sud de la France pour l'essentiel), les gains ne portent à chaque fois que sur quelques dizaines d'emplois supplémentaires (pour 16 zones d'emplois) ou alors quelques centaines (pour 26 zones d'emplois). Cette situation (carte 1), corroborée par la faiblesse du rythme de progression annuelle (carte 2), reste néanmoins une belle performance dans le contexte général, car ces territoires sont aussi concernés par la hausse de la productivité du travail, de même que par celle de la robotique, qui supprime des emplois. L'INSEE ne détaille cependant pas leur ventilation sectorielle.

Carte 1 : Gain et pertes d'emplois industriels par zones d'emplois (1998-2014)

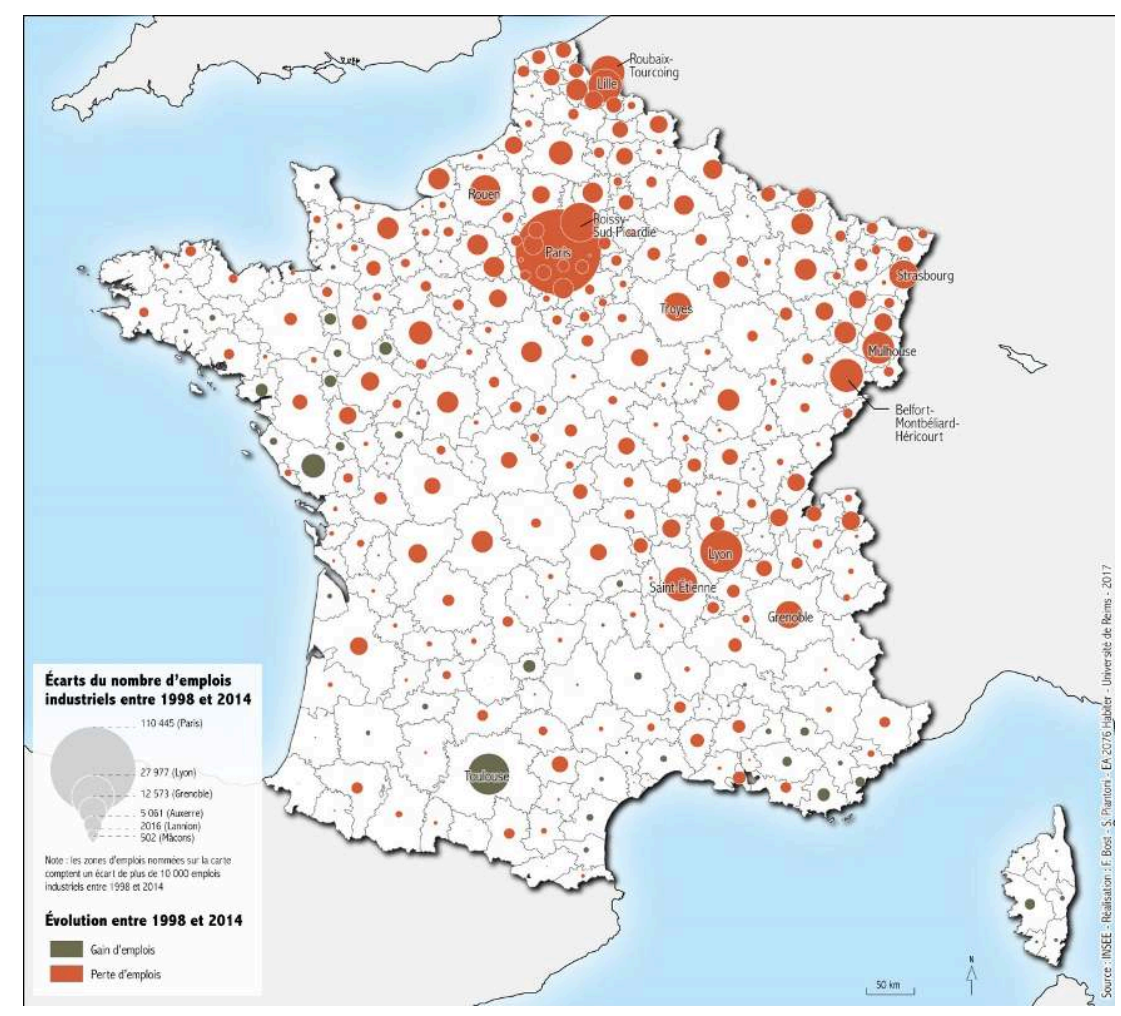


Carte 2 : Evolution de l'emploi industriel par zones d'emplois (1998-2014)

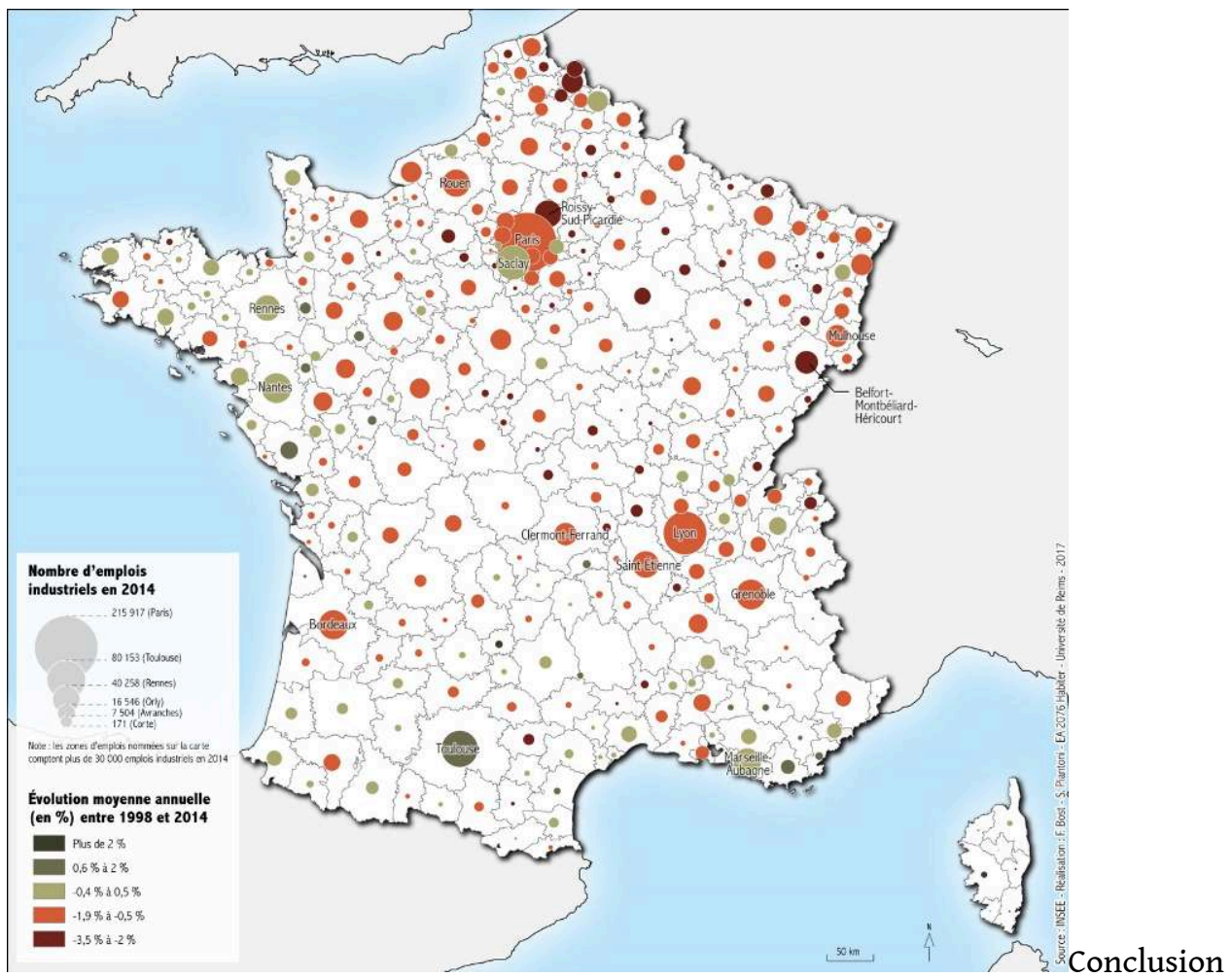

A l'instar de ses voisins européens, la France (à travers ses Pouvoirs publics, ses organisations professionnelles et syndicales, ses acteurs de l'économie, ses observateurs avisés, son opinion publique, etc.) semble avoir pris conscience de l'ampleur et de la gravité de la désindustrialisation qui mine désormais son économie. La crise des années 2007-2008 a joué à cet égard un rôle de révélateur qui a favorisé un électrochoc salutaire. Un certain nombre de signes encourageants allant dans le sens du redressement sont déjà relevés, à l'instar des premières retombées du Crédit d'impôt compétitivité emploi, ou CICE, qui semble avoir joué un rôle bénéfique sur le rétablissement depuis 2015 des marges bénéficiaires des entreprises. Cette condition est en effet indispensable pour faciliter les nouveaux investissements de modernisation d'un appareil de production vieillissant. Alliés à un retour prudent de l'optimisme des chefs d'entreprises depuis 2016-2017, ces signes plus favorables témoignent que la France et ses territoires sont sans doute déjà entrés dans une nouvelle phase de leur histoire industrielle, annonciatrice de changements et de bouleversements plus profonds encore, seuls à même d'inverser la tendance observée. Il est néanmoins beaucoup trop tôt pour en dresser un premier bilan, qui vérifierait l'hypothèse de la réindustrialisation. Mais il est certain que trois dynamiques concomitantes se mettent en place depuis quelques années, avec des effets différenciés selon les secteurs sur l'emploi et les territoires concernés.

53 La première s'organise autour de la recherche du maintien des activités encore présentes sur le territoire national (en particulier dans les vieilles régions industrialisées) et ayant encore un avenir. Sa condition impérative est celle d'un engagement résolu dans un processus de montée en gamme (présenté à bien des égards comme la seule alternative possible sur le modèle des pays de l'Europe du nord, l'Allemagne en premier lieu), mais aussi l'amélioration de leur compétitivité (signature 
par exemple d'accords de compétitivité chez Renault avec les syndicats) et la modernisation rapide des outils de production.

La seconde a suscité beaucoup d'espoirs au cours des années 2014-2015, à savoir la relocalisation depuis l'étranger, à chaque fois que cela est possible, de certaines activités et selon différentes modalités : retour complet d'usines en France, transfert de certaines lignes de production entière, réincorporation d'étapes d'un processus industriel. Mais son élan paraît encore modéré, voire hypothétique, ce qui ne signifie pas qu'il convienne de ne pas s'y intéresser (Bost, 2015). De surcroît, les effets sur l'emploi de ces opérations - souvent très médiatisées en raison des espoirs disproportionnés qu'elles suscitent - s'avèrent faibles, car ces relocalisations sont rendues possibles par un haut niveau d'investissement dans la robotisation et l'automatisation des tâches.

La troisième dynamique est actuellement celle qui fait l'objet de la plus forte attention, puisqu'elle valorise l'avénement d'activités industrielles résolument nouvelles, entièrement fondées sur l'économie numérique et du savoir, en s'appuyant sur la robotisation massive, l'impression 3D appliquée à la production de masse, la réalité augmentée, l'internet des objets (qui va révolutionner le processus de production par la circulation des données numériques, de même que la logistique tout au long de la chaîne d'approvisionnement). Surtout cette révolution annoncée (appelée déjà par certains observateurs "Robolution ») est porteuse de changements et de défis très profonds du monde du travail industriel, car elle sous-entend le recours à des profils et à des compétences nouvelles dans des usines intelligentes et connectées (programme « Usines du futur » lancé en 2013). Autant de bouleversements majeurs à venir qui font penser que l'industrie sait aussi se réinventer en France.

\section{BIBLIOGRAPHIE}

Askenazy Philippe, 2012, « Faut-il craindre la désindustrialisation ? ", Problèmes économiques, $\mathrm{n}^{\circ}$ 3040, p. 35-40.

Baudelle Guy, Fache Jacques, 2014, Les mutations des systèmes productifs en France, Rennes, Presses universitaires de Rennes, $373 \mathrm{p}$.

Bell Daniel, 1976, The Coming of Post-Industrial Society. A Venture in Social Forecasting, New York, Basic Books, 507 p. (trad. française : Vers la société post-industrielle, Paris, Robert Laffont).

Blanchet Max, 2013, L'industrie France décomplexée. Choisir nos batailles et créer un modèle industriel à la française, Paris, Lignes de repères, $192 \mathrm{p}$.

Bost François, 2015, « Les relocalisations industrielles en France. Enjeux, limites et opportunités nouvelles », Bulletin de l'Association des Géographes Français, décembre, p. 480-494.

Bost François, 2014, La France : mutations des systèmes productifs, Paris, Armand Colin, 253 p.

Bost François, 2012, Entreprises et territoires à l'épreuve de la mondialisation de l'économie, Université de Paris-Ouest-Nanterre-La Défense, Habilitation à diriger des recherches, $369 \mathrm{p}$. 
Carroué Laurent, 2013, La France. Les mutations des systèmes productifs, Paris, Armand Colin, 235 p.

Carroué Laurent, 2012, «Crises et basculements des équilibres mondiaux : l'entrée dans le XXI ${ }^{\mathrm{e}}$ siècle », Images économique du monde 2013, Paris, Armand Colin, p. 8-27.

Colletis Gabriel, 2012, L'urgence industrielle. Paris, Le bord de l'eau, 2012, 200 p.

Cohen Elie, 2013, Crise ou changement de modèle ? Paris, La documentation Française, 106 p.

Cohen Elie, Buigues Pierre-André, 2014, Le décrochage industriel, Paris, Fayard, 439 p.

Cowie Jefferson, Heathcott Joseph, Bluestone Barry, 2003, Beyond the Ruins: The Meanings of

Deindustrialization, Ilr Press Books, 292 p.

Davezies Laurent, 2012, La crise qui vient, Paris, Le Seuil, 128 p.

Demmou Lilas, 2010, La désindustrialisation en France, Direction générale du trésor et de la politique économique (DGTPE), document de travail, numéro 2010/01, 49 p.

Demmou Lilas, 2010, « Le recul de l'emploi industriel en France entre 1980 et 2007. Ampleur et principaux déterminants : un état des lieux », Économie \& Statistique, ${ }^{\circ} 438-440$.

Dugot Philippe, Thuillier Guy., 2014, France : les mutations des systèmes productifs, Toulouse, Presses universitaires de du Mirail, $455 \mathrm{p}$.

Feiertag Olivier, 2011, « L'économie française de 1914 à nos jours », Paris, La Documentation photographique, $64 \mathrm{p}$.

Fontagné Lionel, Lorenzi Jean-Hervé, 2005, Désindustrialisation, délocalisations, Paris, La documentation Française, 397 p.

Fourastié Jean, 1949, Le Grand Espoir du XX $X^{e}$ siècle. Progrès technique, progrès économique, progrès social, Paris, Presses universitaires de France, $224 \mathrm{p}$.

Gallois Louis, 2012, Pacte pour la compétitivité de l'industrie française, Paris, La documentation Française, $67 \mathrm{p}$.

Gilly Jean-Pierre, Wallet Frédéric, 2001, « Forms of Proximity Local Governance and the Dynamics of Local Economic Spaces: The Case of Industrial Conversion Processes », International Journal of Urban and Regional Research, vol. 25, $n^{\circ}$ 3, p. 553-570.

Giraud Pierre-Noël, Weil Thierry., 2013, L'industrie française décroche-t-elle ? Paris, La documentation Française, 190 p.

Le Blanc Gilles, 2012, « Dynamiques des territoires industriels. Essai de prospective dans le cadre de Territoires $2040 »$, Futuribles, n 385, p. 24

Le Blanc Gilles, 2011, «Les espaces de la dynamique industrielle », in Territoires 2040, Paris DATAR, revue en ligne $\mathrm{n}^{\circ}$ 3, $170 \mathrm{p}$. URL : http://territoires2040.datar.gouv.fr/spip.php?article86\&revue=1

Le Blanc Gilles, 2011, « Des systèmes spatiaux en perspective », in Territoires 2040, DATAR, revue en ligne $\mathrm{n}^{\circ} 3,170 \mathrm{p}$.

Le Blanc Gilles, 2005, L'industrie dans l'économie française (1978-2003) : une étude comparée, Paris, Institut de l'entreprise, avril, $112 \mathrm{p}$.

Lorenzi Jean-Hervé, 2014, Le choc de la désindustrialisation, in Un monde de violences. L'économie mondiale, 2015-2013, Paris, Eyrolles, p. 109-145.

Martin Ron., Rowthorn Bob., 1986, The Geography of Deindustrialization, Macmillan Publishers Limited, $395 \mathrm{p}$. 
Messaoudi Dalila, 2015, « Les territoires français de l'automobile à l'épreuve des mutations des systèmes productifs ", Bulletin de l'association des géographes français, $n^{\circ}$ 4, p. 495-510.

Messaoudi Dalila, 2012, Les enjeux géoéconomiques, industriels et territoriaux de la délocalisation des entreprises Françaises, Paris-Ouest-Nanterre La Défense, Thèse de doctorat de $\mathrm{III}^{\mathrm{e}}$ cycle en géographie, $459 \mathrm{p}$.

Messaoudi Dalila, 2011, « Les territoires de l'industrie du textile et de l'habillement à l'épreuve des délocalisations ", Bulletin de l'association des géographes français, nº 3, p. 137-149.

Nesta Lionel, 2010, « Désindustrialisation ou mutation industrielle? ", Economie et statistique, Vol. $\mathrm{n}^{\circ} 438-440$, p. 297-301.

Roustan Max, 2004, La désindustrialisation du territoire : mythe ou réalité ?, Rapport d'information à l'Assemblée nationale, $n^{\circ} 1625$ (XII législature), mai, $219 \mathrm{p}$.

Rowthorn Robert, Ramaswamy Ramana., 1997, « Desindustrialization. Its causes and implications ", IMF, Economic issues, $\mathrm{n}^{\circ} 10$.

Touraine Alain, 1969, La société post-industrielle. Naissance d'une société, Paris, Denoël, 315 p.

Veltz Pierre, 2017, La société hyper-industrielle. Le nouveau capitalisme productif, Paris, Seuil, 120 p.

Veltz Pierre, Weil Thierry (dir), 2015, L'industrie, notre avenir, Paris, Eyrolles, colloque de Cerisy, $343 \mathrm{p}$.

Verlet Patrick, 1997, La Révolution industrielle, Paris, Folio histoire, Gallimard, 543 p.

Woessner Raymond, 2015, « La territorialisation par l'industrie automobile : proposition pour la compréhension du phénomène par une entrée systématique », Bulletin de l'association des géographes français, $\mathrm{n}^{\circ}$ 4, p. 466-479.

\section{NOTES}

1. En nombre de véhicules assemblés sur le sol national.

2. Pour le détail complet des communes: https://www.insee.fr/fr/metadonnees/cog/zone-demploi/ZE20101101-paris

\section{RÉSUMÉS}

Comme tous les autres pays européens, la France connait un mouvement de désindustrialisation rapide. Celui-ci est devenu encore plus flagrant à la faveur de la crise financière et économique apparue à partir des années 2007-2008. Si désormais la question de la désindustrialisation occupe largement le champ médiatique, au point de commencer à inquiéter le politique, les acteurs économiques et l'opinion publique, comment ce phénomène se mesure-t-il objectivement ? Quels sont ses indicateurs et sont-ils pertinents pour l'analyser? Cet article entend donc faire le point sur la perception, la mesure et la signification réelle des chiffres de la désindustrialisation. Un bilan par zones d'emplois terminera l'analyse pour rendre compte de situations très contrastées à l'échelle nationale. 
Like all the other European countries, France knows a fast movement of deindustrialization.This one became even more obvious owing to the financial crisis and economic appeared as from the years 2007-2008. If this question of deindustrialization largely occupies the media field, at the point to start to worry the policy, the economic actors and the public opinion, how this phenomenon measured is objectively? What are its indicators and are they relevant to analyze it? This article intends to take stock of perception, the measurement and the real significance of the figures of deindustrialization. An assessment by zones of employment will finish the analysis to give an account of very contrasted situations to the national scale.

Frankreich kennt gerade, wie alle anderen europäischen Ländern, eine Tendenz zu einer schnellen Desindustrialisierung. Diese wurde noch mehr offensichtlich durch die Finanz- und Wirtschaftskrise seit den Jahren 2007-2008. Wenn nun die Frage der Desindustrialisierung die Medien weitgehend besetzt, so dass die Politik, die Wirtschatsakteure und die Offentlichkeit sich jetzt Gedanken machen, dann wie kann man dieses Phänomen objektiv messen? Was sind die Indikatoren und sind sie relevant zur Analyse ? Das Ziel dieses Artikels ist eine Bestandaufnahme über die Wahrnehmung, die Messung und die wahre Bedeutung der Desindustrialisierungszahlen zu geben. Die Analyse erfolgt durch eine Bewertung jeder Arbeitsplatz, um Situationen sehr kontrastreiche auf nationaler Ebene zu berücksichtigen.

\section{INDEX}

Schlüsselwörter : Wirtschaft, Unternehmen, Deindustrialisierung, Globalisierung

Keywords : economy, enterprises, globalization, deindustrialization

Mots-clés : économie, entreprises, désindustrialisation, mondialisation

\section{AUTEURS}

\section{FRANÇOIS BOST}

Professeur des universités, Université de Reims Champagne-Ardenne (URCA), 57 rue Pierre Taittinger, 51096 REIMS Cedex <francois.bost@univ-reims.fr>

\section{DALILA MESSAOUDI}

Maître de conférences, Université de Versailles Saint-Quentin-en-Yvelines (UVSQ), 47 boulevard Vauban, 78047 Guyancourt Cedex <dalila.messaoudi@uvsq.fr> 